Leonard H. Calabrese, DO

Department of Rheumatic and Immunologic Diseases, Orthopedic \& Rheumatologic Institute,

Cleveland Clinic
Tiphaine Lenfant, MD

Department of Rheumatic and Immunologic Diseases, Orthopedic \& Rheumatologic Institute Department of Infectious Disease, Cleveland Clinic; Assistance Publique des Hôpitaux de Paris, Université de Paris; Hôpital européen Georges Pompidou, Service de médecine interne, Paris, France
Cassandra Calabrese, DO

Department of Rheumatic and Immunologic Diseases,

Orthopedic \& Rheumatologic Institute, and

Department of Infectious Disease, Cleveland Clinic

\title{
Cytokine storm release syndrome and the prospects for immunotherapy with COVID-19, part 3: The role of GM-CSF
}

\section{Posted August 10, 2020}

\section{ABSTRACT}

Granulocyte-macrophage colony-stimulating factor (GM-CSF) has been used experimentally in patients with acute respiratory distress syndrome. Recombinant GMCSF administered by direct inhalation is currently being studied in a cohort of patients with advanced COVID-19.

\section{INTRODUCTION}

In the first two parts of this series, we focused on the basic immunobiology of severe COVID-19 disease and the role of inflammatory cytokines in driving respiratory damage, coagulopathy, end-organ failure, and death, which is idealized in Figure 1.,2 We know that $90 \%$ of patients with COVID-19 recover, but we are also aware that in about $10 \%$ of patients the disease is progressive, and that this is heavily influenced by a growing number of risk factors, including attendant cardiovascular disease, obesity, hypertension, and age. This stage 3 of COVID-19, when the disease may rapidly progress to fatality, has been continuously elucidated and referred to as "cytokine storm," given the presence of elevated levels of inflammatory cytokines and chemokines including interleukin 1 (IL-1), IL-6, tumor necrosis factor (TNF), granulocytemacrophage colony-stimulating factor (GM-CSF), gamma interferon, and monocyte chemotactic protein-1 (MCP-1). ${ }^{2}$ Furthermore, quantitative studies of these biomarkers have demonstrated that some may discriminate mild self-limiting forms of COVID19 from severe progressive forms of the disease. ${ }^{3}$ But

The statements and opinions expressed in COVID-19 Curbside Consults are based on experience and the available literature as of the date posted. While we try to regularly update this content, any offered recommendations cannot be substituted for the clinical judgment of clinicians caring for individual patients.

doi:10.3949/ccjm.87a.ccc057 still lacking is an understanding of the precise choreography of cytokine storm and of which cytokine or cytokines are upstream drivers vs those that may be late-stage effectors or amplifiers. While IL-6 and IL-1 are both logical therapeutic targets and the preliminary data from uncontrolled studies have shown promise, inhibition has not been curative and is not without real safety concerns. This Curbside Consult will focus on another cytokine of growing interest, GM-CSF.

\section{BASIC IMMUNOLOGY OF GM-CSF}

GM-CSF is a complex cytokine and a member of the colony-stimulating superfamily. While it does have a role as a growth factor for myeloid cells, this is considered a lesser function compared with other cytokines such a granulocyte-stimulating colony factor (G-CSF) and macrophage-stimulating colony factor (M-CSF), and GM-CSF is now considered a central player in the integrated immune response and a central mediator of tissue inflammation. ${ }^{4,5}$

GM-CSF is produced by both hematopoietic (eg, $\mathrm{T}$ cells, B cells, macrophages, monocytes) and some viscerosomatic cells (type II alveolar epithelial cells, endothelial cells, fibroblasts) and can activate cells through the dimeric GM-CSF receptor expressed primarily on monocytes and macrophages but also on dendritic cells and other cells of the innate immune system (Figure 2A).,46 GM-CSF mediates its effects by signaling through the JAK-STAT pathway, ${ }^{7}$ a pathway inhibited by currently available therapeutics including tofacitinib and baricitinib. During inflammation GM-CSF serves two important roles (Figure 2B). First, it functions to polarize mature myeloid cells into a pro-inflammatory phenotype capable of secreting other inflammatory cytokines such as IL-1, IL-6, and TNF, as well as a variety of chemokines 
Stage 1

Asymptomatic

Immune response over time:

Self-limiting in $\mathbf{8 0} \%$

Severe in $15 \%-20 \%$

Fatal in $1 \%-2 \%$

Innate immune
activation

Viral engagement of PAMPs

Low type 1 IFN
Stage 2

Nonsevere symptomatic
Adaptive immune
activation

Generation of specific antibodies and T-cell response

Release of DAMPs
Stage 3

Severe respiratoryinflammatory

Cytokine release syndrome

IL-1, IL-6, TNF, GM-CSF,

IFN-gamma, others

Coagulopathy

Complement

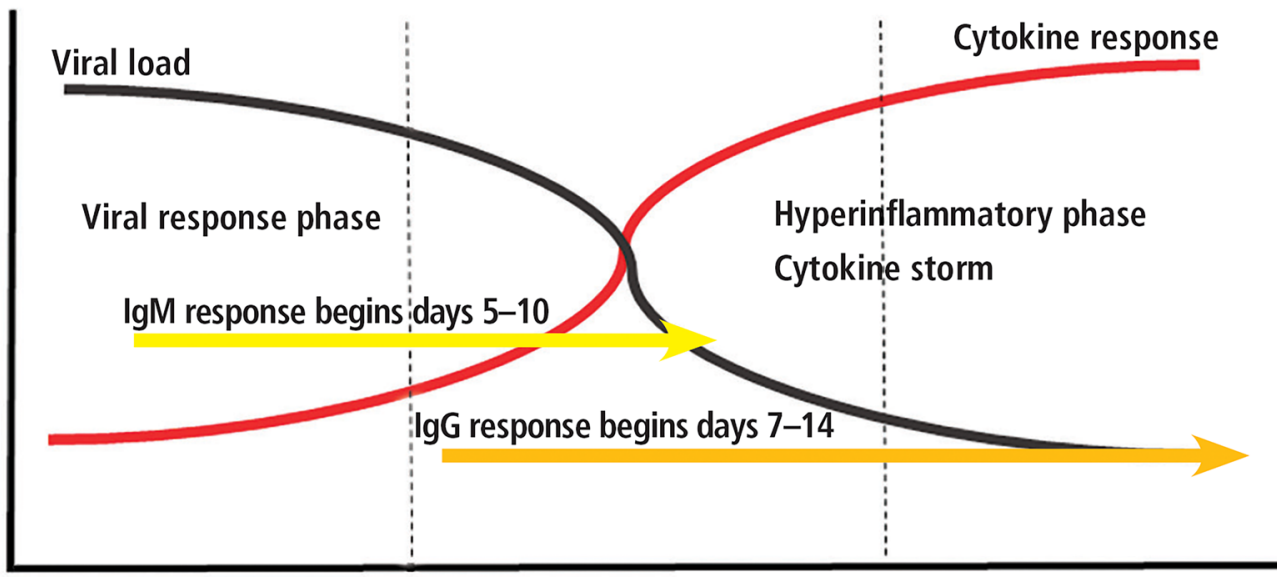

Time course

DAMPs = damage-associated molecular patterns; GM-CSF = granulocyte macrophage colony-stimulating factor; IFN = interferon; IgM = immunoglobulin M; IL-1 = interleukin 1; IL-6 = interleukin 6; PAMPs = pathogen-associated molecular patterns; TNF = tumor necrosis factor

Figure 1. Three stages of COVID-19 disease.

governing trafficking of hematopoietic cells to areas of inflammation. GM-CSF also serves to activate dendritic cells to prime $T$ cells during antigen-specific responses and thus links the myeloid compartment and pathogenic T cells (TH-1 and TH-17) in a positive feedback loop, capable of propagating inflammation and tissue injury.

Of particular relevance and importance regarding the immunobiology of GM-CSF in COVID-19 disease is its complex role in lung homeostasis and inflammation. In the healthy lung, GM-CSF has a critical role for maintaining the maturation and function of alveolar macrophages and surfactant metabolism ${ }^{5}$ and is required to maintain pulmonary function, as well as contributing to lung sentinel cell-mediated immunity. GM-CSF also appears central in driving inflammation locally and systemically, ${ }^{5}$ and experimental models of acute lung injury support this hypothesis, demonstrating that resident alveolar macrophages secrete a variety of inflammatory cytokines that lead to the influx of innate cells including neutrophils, fur- ther amplifying the activation of alveolar epithelial cells and tissue damage. ${ }^{8}$

The capacity of GM-CSF to amplify inflammatory response within the lung and its systemic effects, mediated by linking the release of upstream inflammatory cytokines such as IL-1, IL-6, and TNF across monocytes and macrophages and activated $T$ cells in a positive feedback loop, highlight its potential importance in driving systemic inflammation and disease. However, as noted above, adding to the complexity is that GM-CSF is also a critical cytokine for healthy pulmonary function and is necessary for the maturation and maintenance of alveolar macrophages; and in some experimental models, it confers resistance to viral respiratory balance, underscoring that all putative inflammatory cytokines also play roles in integrated host defense. ${ }^{4}$

The central role of GM-CSF in the inflammatory response and because GM-CSF appears to be upstream of other key inflammatory cytokines invites targeting strategies against it in effort to 
A

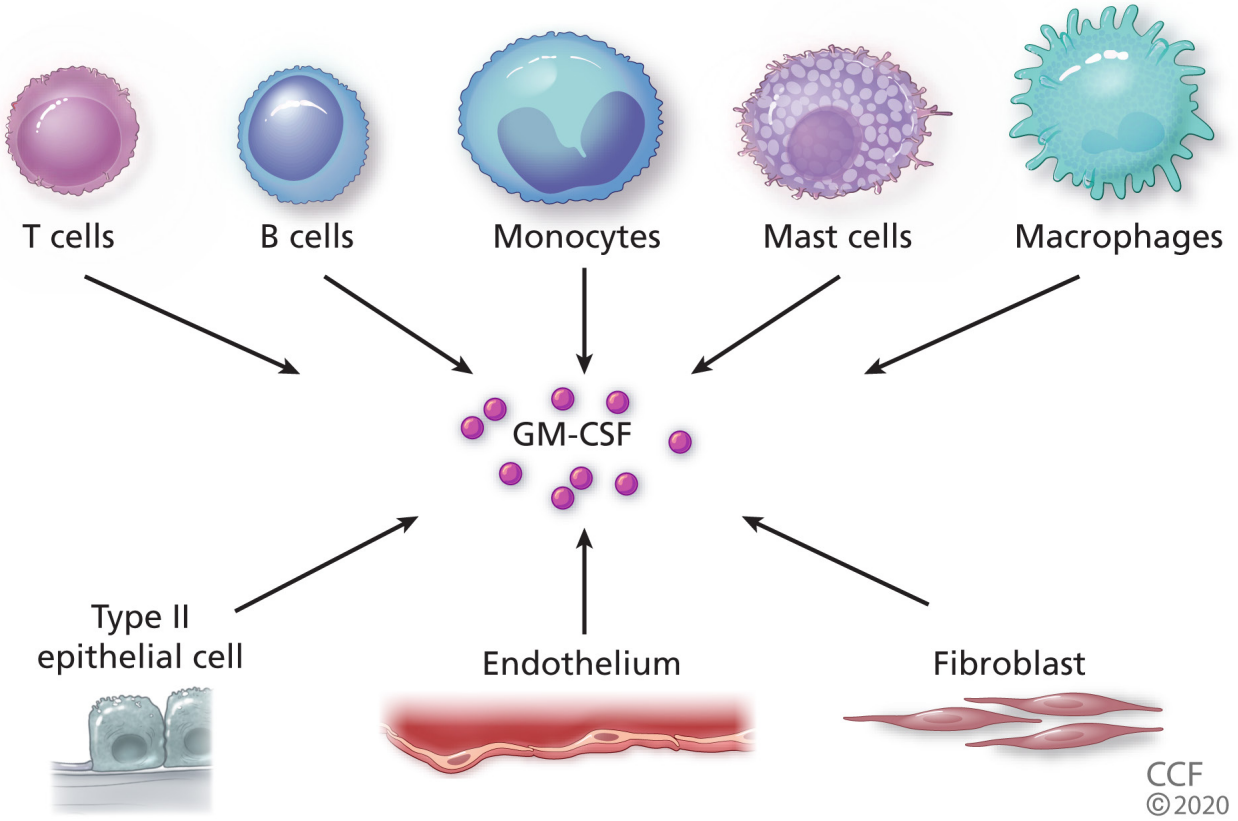

B

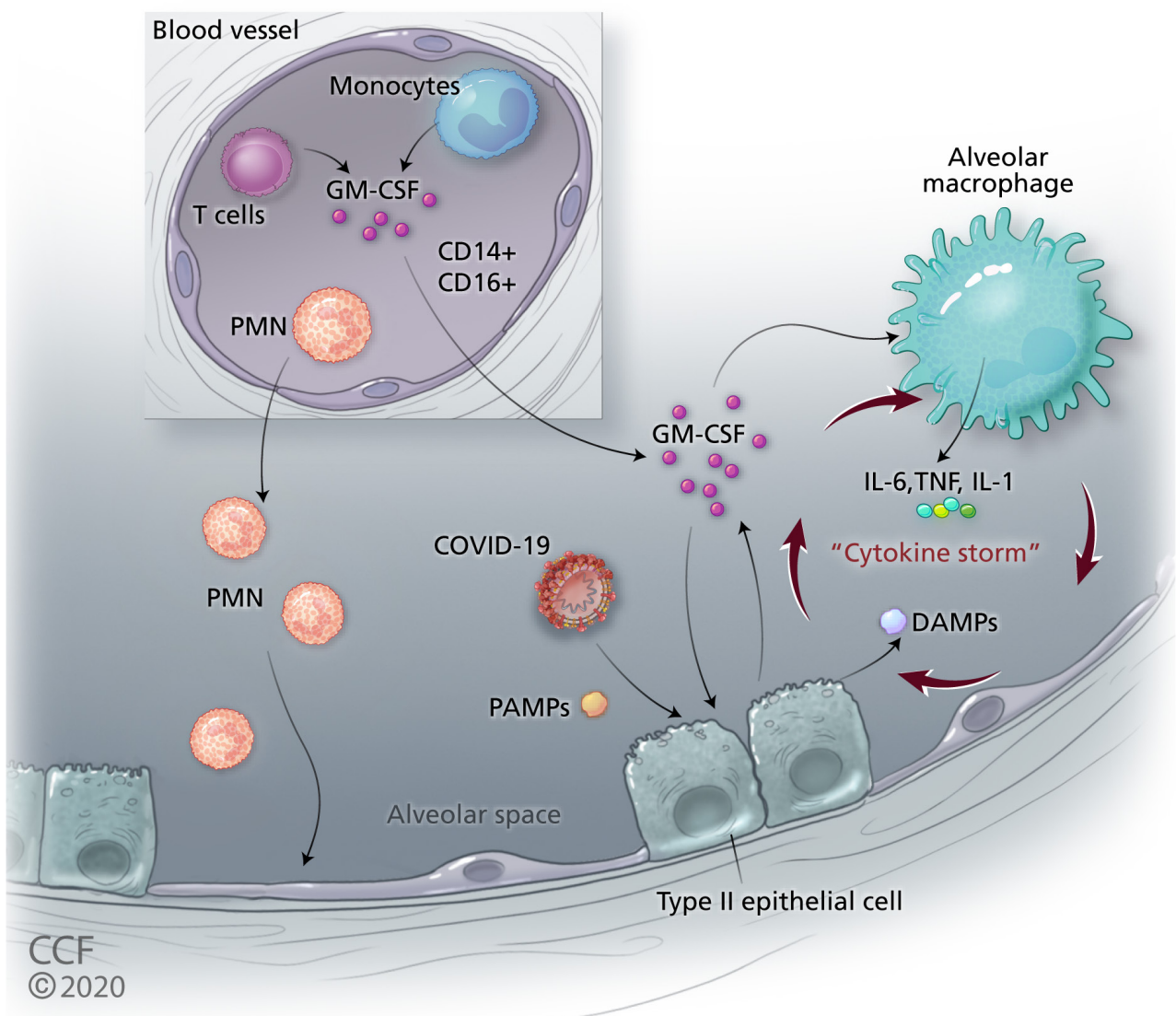

Figure 2. (A) Granulocyte-macrophage colony-stimulating factor (GM-CSF) is produced by hematopoietic cells (top) with inflammatory monocytes and activated TH1 and $\mathrm{TH} 17$ being among the richest sources, as well as by a variety of viscerosomatic cells including Type 2 pulmonary epithelial cells. (B) A schematic where GM-CSF plays a central role in alveolar damage in COVID-19. Depicted is the virus (SARS-CoV-2) infecting alveolar epithelial cells and upregulating inflammatory cytokine production including GM-CSF. Excess GM-CSF in concert with other cytokines may further upregulate activated alveolar macrophages, which further stimulates release of GM-CSF. Activated T cells and polarized monocytes from peripheral blood may now contribute locally and systemically by responding with release of downstream cytokines such as interleukin 1 (IL-1), tumor necrosis factor (TNF), IL-6, and chemokines, which may then attract polymorphonuclear leukocytes causing further tissue damage and release of damage-associated molecular patterns (DAMPS) further driving inflammatory signaling. 
TABLE 1

Overview of anti-GM-CSF drugs, mechanisms, and studies

\begin{tabular}{|c|c|c|c|}
\hline Mechanism of action & Drug & Study population & Phase \\
\hline \multirow{5}{*}{$\begin{array}{l}\text { Humanized immunoglobulin G1 } \\
\text { (IgG1) monoclonal antibody target- } \\
\text { ing granulocyte-macrophage colony- } \\
\text { stimulating factor (GM-CSF) }\end{array}$} & TJ003234 & $\begin{array}{l}\text { Healthy individuals } \\
\text { COVID-19 }\end{array}$ & $\begin{array}{c}1 \\
1 \mathrm{~b} / 2\end{array}$ \\
\hline & Gimsilumab & $\begin{array}{l}\text { Ankylosing spondylitis } \\
\text { COVID-19 }\end{array}$ & $\begin{array}{l}1 \\
2\end{array}$ \\
\hline & Lenzilumab & $\begin{array}{l}\text { Chronic myelomonocytic leukemia } \\
\text { Relapsed or refractory large B-cell lymphoma } \\
\text { COVID-19 }\end{array}$ & $\begin{array}{c}1 \\
1 / 2 \\
3\end{array}$ \\
\hline & Otilimab & $\begin{array}{l}\text { Rheumatoid arthritis } \\
\text { Inflammatory arthritis } \\
\text { COVID-19 }\end{array}$ & $\begin{array}{c}2 b \\
2 \\
2\end{array}$ \\
\hline & Namilumab & $\begin{array}{l}\text { Rheumatoid arthritis } \\
\text { Chronic plaque psoriasis } \\
\text { Spondyloarthritis }\end{array}$ & $\begin{array}{l}2 \\
2 \\
2\end{array}$ \\
\hline $\begin{array}{l}\text { Humanized lgG4 monoclonal } \\
\text { antibody targeting GM-CSF receptor } \\
\text { alpha }\end{array}$ & Mavrilimumab & $\begin{array}{l}\text { Rheumatoid arthritis } \\
\text { Giant cell arthritis } \\
\text { COVID-19 }\end{array}$ & $\begin{array}{c}2 b \\
2 \\
2\end{array}$ \\
\hline $\begin{array}{l}\text { Betac-receptor-specific, fully human } \\
\text { IgG4 monoclonal antibody (inhibitor } \\
\text { of IL-3-, GM-CSF-, and IL-5-mediat- } \\
\text { ed functions) }\end{array}$ & CSL311 & Asthma & 1 \\
\hline
\end{tabular}

down-modulate states of hypercytokinemia. GMCSF levels are generally extremely low or undetectable in healthy individuals and are detectable in blood of patients with inflammatory manifestations of COVID-19, ${ }^{9}$ and CD14+CD16+ monocytes, a rich source of GM-CSF, are expanded in such patients as well. ${ }^{10}$ As noted in Table 1, GM-CSF targeting is actively being investigated in a variety of autoimmune diseases and has been successfully studied in a mouse model of cytokine release from CAR-T cells. ${ }^{11}$ Based on these data, investigation of targeting GMCSF in COVID-19 has commenced.

\section{TARGETING GM-CSF IN AUTOIMMUNE AND INFLAMMATORY DISEASES AND COVID-19}

There is a rich pipeline of biologic therapeutics that target GM-CSF directly or that targeting the GMCSF receptor, and none are currently approved for any indication, though they are under investigation in many conditions, including rheumatoid arthritis, spondyloarthritis, giant cell arteritis, psoriasis, and certain malignancies (Table 1 ). To date, there are 8 clinical trials of varying design and size (Table 2) focused mainly on patients with advancing COVID19 that does not require mechanical ventilation.

In the most detailed clinical report to date, De Luca et $\mathrm{al}^{12}$ described a single-center, nonrandomized, prospective cohort study in which 13 non-mechanically ventilated patients with pneumonia, hypoxia, and signs of systemic inflammation were treated with the anti-GM-CSF receptor mavrilimumab given as single intravenous dose. They compared this group to a cohort of 26 patients treated simultaneously with similar baseline characteristics receiving the same standard of care without mavrilimumab. At 28 days, no patients in the anti-GM-CSF group died, whereas $7(27 \%)$ of 26 in the control group died. The agent was well tolerated with no infusion reactions.

This study, while encouraging, is cautionary on the basis of its design and the inherent risks of unobserved confounders. Robust trials are under way and will hopefully provide more definitive evidence (Table 2). A second single-center study from Mayo Clinic released but not yet peer-reviewed also described impressive results. ${ }^{13}$ In this observational noncontrolled study, 12 patients with COVID-19 pneumonia 


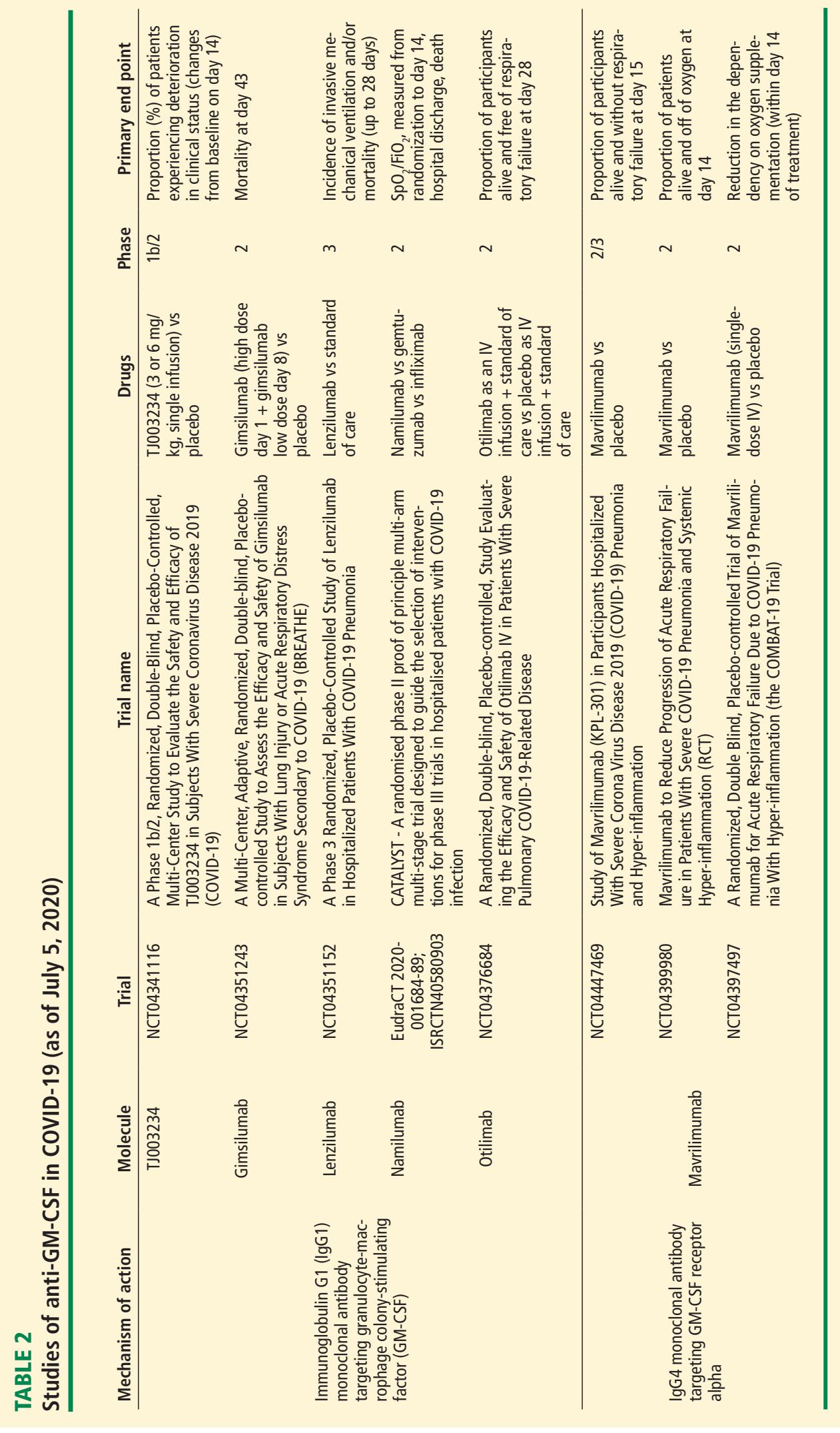


and risk factors for poor outcome were treated with 3 doses of lenzilumab, and clinical improvement was noted in $92 \%$ with a median time-to-discharge of 5 days. Detailed analysis of inflammatory biomarkers and cytokines also improved, and there were no treatment-related adverse events. In summary, while encouraging, these data make completion of randomized trials even more urgent.

\section{SAFETY CONCERNS}

Targeting GM-CSF carries with it a number of safety concerns derived from both preclinical modeling and human disease. GM-CSF is an integral part of the integrated immune response and, as noted above, is important in maintaining defense against infections. Thus, any use in COVID-19 must weigh this balance. Fortunately, in the collective clinical experience reported across many trials, targeting GM-CSF has been well tolerated with no major safety signals. ${ }^{5}$ Of high concern in all GM-CSF trials is the specter of pulmonary alveolar proteinosis, a rare disease characterized by accumulation of proteinaceous material in the lungs; some patients with this disease have autoantibodies directed against GM-CSF.14 This toxicity has been monitored as an event of special interest in clinical trials for autoimmune disease, and has not been observed to date.

Finally, a recurrent theme with all clinical trials in COVID-19 inflammatory disease is timing. Giving an immunomodulator too early in the course of the infection will compromise an evolving adaptive immune response and may theoretically prolong the disease and compromise the outcome. ${ }^{2,8}$ At the moment there is no definitive single biomarker or set of biomarkers capable of identifying the optimal timing of such interventions. How this therapy will fare used singly or in combination with antiviral therapy or glucocorticoids, which are at the moment in ascendency for treatment of COVID-19, will require larger and more carefully conducted trials.

\section{RATIONALE FOR GIVING GM-CSF IN COVID-19}

Consistent with the complex immunobiology of GMCSF is a seemingly contradictory strategy of administering GM-CSF to patients with late-stage COVID-19 disease. This theory is based on the role of GM-CSF previously noted including its centrality in maintaining critical lung function and local anti-microbial defense. ${ }^{5}$ Numerous preclinical models appear to support this concept, and GM-CSF, an approved biologic agent, has been utilized experimentally in patients with acute respiratory distress syndrome. A trial of recombinant GM-CSF administered by direct inhalation in a cohort of patients with advanced COVID19 (NCT04326920) is under way.

These divergent strategies underscores our incomplete understanding of the immunobiology of COVID-19 and its most sinister clinical outcome, acute respiratory distress syndrome, and the need to carefully study such strategies in controlled settings.

\section{REFERENCES}

1. Calabrese C, Rajendram P, Sacha G, Calabrese L. Practical aspects of targeting IL-6 in COVID-19 disease. Cleve Clin J Med 2020. [Epub ahead of print] doi:10.3949/ccjm. 87 a.ccc018

2. Calabrese LH. Cytokine storm and the prospects for immunotherapy with COVID-19. Cleve Clin J Med 2020; 87(7):389-393. doi:10.3949/ ccjm.87a.ccc008

3. Wang F, Hou H, Luo $\mathrm{Y}$, et al. The laboratory tests and host immunity of COVID-19 patients with different severity of illness. JCI Insight 2020; 5(10):e137799. doi:10.1172/jci.insight.137799

4. Becher B, Tugues S, Greter M. GM-CSF: from growth factor to central mediator of tissue inflammation. Immunity 2016; 45(5):963973. doi:10.1016/j.immuni.2016.10.026

5. Lang FM, Lee KM, Teijaro JR, Becher B, Hamilton JA. GM-CSFbased treatments in COVID-19: reconciling opposing therapeutic approaches. Nat Rev Immunol 2020; 20(8):507-514. doi:10.1038/ s41577-020-0357-7

6. Lotfi N, Thome R, Rezaei N, et al. Roles of GM-CSF in the pathogenesis of autoimmune diseases: an update. Front Immunol 2019; 10:1265. doi:10.3389/fimmu.2019.01265

7. Wicks IP, Roberts AW. Targeting GM-CSF in inflammatory diseases. Nat Rev Rheumatol 2016; 12(1):37-48. doi:10.1038/ nrrheum.2015.161

8. Mehta P, Porter JC, Manson JJ, et al. Therapeutic blockade of granulocyte macrophage colony-stimulating factor in COVID19-associated hyperinflammation: challenges and opportunities. Lancet Respir Med 2020. [Epub ahead of print] doi:10.1016/ S2213-2600(20)30267-8

9. Huang C, Wang $Y$, Li $X$, et al. Clinical features of patients infected with 2019 novel coronavirus in Wuhan, China. Lancet 2020; 395(10223):497-506. doi:10.1016/S0140-6736(20)30183-5

10. Zhou $Y$, Fu B, Zheng $X$, et al. Aberrant pathogenic GM-CSF+ T cells and inflammatory CD14+CD16+ monocytes in severe pulmonary syndrome patients of a new coronavirus. bioRxiv 2020 . doi:10.1101/2020.02.12.945576

11. Sterner RM, Sakemura R, Cox MJ, et al. GM-CSF inhibition reduces cytokine release syndrome and neuroinflammation but enhances CAR-T cell function in xenografts. Blood 2019; 133(7):697-709. doi:10.1182/blood-2018-10-881722

12. DeLuca G, Cavalli G, Caampochiaro C, et al. GM-CSF blockade with mavrilimumab in severe COVID-19 pneumonia and systemic hyperinflammation: a single center, prospective cohort study. Lancet Rheumatol 2020. [Epub ahead of print] doi:10.1016/ S2665-9913(20)30170-3

13. Temesgen $\mathbf{Z}$, Assi $\mathbf{M}$, Vergidis $\mathbf{P}$, et al. First clinical use of lenzilumab to neutralize GM-CSF in patients with severe COVID-19 pneumonia. medRxiv 2020. [Epub ahead of print] doi:10.1101/2020.06.08.20125369

14. Trapnell BC, Nakata K, Bonella F, et al. Pulmonary alveolar proteinosis. Nat Rev Dis Primers 2019; 5(1):16. doi:10.1038/s41572-019-0066-3

Correspondence: Leonard H. Calabrese, DO, Department of Rheumatic and Immunologic Diseases, A50, Cleveland Clinic, 9500 Euclid Avenue, Cleveland, $\mathrm{OH}$ 44195; calabrl@ccf.org 